\title{
CENTRO HISTÓRICO DE LA CIUDAD DE JÁCHAL - SAN JUAN - ARGENTINA: LINEAMIENTOS PARA LA CONSERVACIÓN DE SU PATRIMONIO CULTURAL
}

\author{
Historical Center of Jáchal city -San Juan - Argentina: \\ guidelines for the conservation of its cultural heritage
}

Ana Valeria Pochi Dorazio

Universidad Nacional de San Juan, Argentina

anapochi@live.com.ar

Eliana Gabriela Testa

Universidad Nacional de San Juan, Argentina

elianatesta@yahoo.com.ar

María Emilia Erostarbe Suárez

Universidad Nacional de San Juan, Argentina

emiliaerostarbe@gmail.com

María Rosa Plana

Universidad Nacional de San Juan, Argentina

planamariarosa@gmail.com

Recibido: 27-08-2017

Aprobado: 04-10-2017

\section{RESUMEN}

Ana Valeria Pochi Dorazio es arquitecta, docente e investigadora en FAUD, UNSJ, IRPHA (Instituto Regional de Planeamiento y Hábitat). Codirectora de proyectos de investigación. Es profesora adjunta y doctoranda en el Doctorado de Arquitectura y Urbanismo de la misma universidad. Becas CICYTCA UNSJ Iniciación y Perfeccionamiento.
El presente trabajo pertenece al "Plan de Manejo del Centro Histórico de la ciudad de Jáchal", San Juan, Argentina, para su conservación, revitalización, recuperación y puesta en valor ${ }^{1}$. Se partió del relevamiento de cada parcela del centro histórico con planillas preestablecidas, donde se tuvieron en cuenta las variables más destacadas como el tipo de construcción, estilo, estado, valor patrimonial y uso del suelo. Las etapas se llevaron a cabo en fases: Relevamiento in situ, Digitalización de datos y Grilla interpretación de datos.

Como resultado final se obtuvo una herramienta de análisis que combina perfiles urbanos fotográficos y la gráfica de datos del casco histórico que posibilitaron los diagnósticos correspondientes de la situación actual de la zona analizada y poder realizar los lineamientos normativos para la conservación y puesta en valor del Centro Histórico de Jáchal.

\footnotetext{
${ }^{1}$ Este trabajo se enmarca en un Convenio entre el Ministerio de Cultura de la Provincia de San Juan y la Universidad Nacional de San Juan, Facultad de Arquitectura, Urbanismo y Diseño, Instituto Regional de Planeamiento y Hábitat, Área Patrimonio, Tecnología y Desarrollo - Argentina.
} 
Elina Gabriela Testa, es arquitecta, docente e investigadora en FAUD, UNSJ, IRPHA (Instituto Regional de Planeamiento y Hábitat). Directora del Proyecto Museo Histórico Etnográfico Itálico "Ausonia". Doctoranda en el Doctorado de Arquitectura y Urbanismo de la misma universidad. Becas CONICET Tipo I y CICYTCA UNSJ Iniciación.

Maria Emilia Erostarbe Suárez es licenciada en Turismo -FFHA, UNSJ. Docente e investigadora FAUS, UNSJ, IRPHA (Instituto Regional de Planeamiento y Hábitat). Maestranda en Maestría en Gestión Turística del Patrimonio, Especialización en Paisajes Culturales. Doctoranda en el Doctorado en Ciencias Scoiales, de la misma universidad. Becaria doctoral CONICET, Temas Estratégicos.

María Rosa Plana es doctora en arquitectura, investigadora $y$ directora de proyectos IRPHA (Instituto Regional de Planeamiento y Hábitat). Mg. En Conservación, rehabilitación, reciclaje, mantenimiento y restauración del Patrimonio Edificado. CICOP. Socia Fundadora ACODEPAS. Ex Delegada en la Provincia de San Juan de la CNMMLH, Secretaría de Cultura, Presidencia de Nación.

conjunto de la de profesionales investigadores universitarios y referentes gubernamentales se planteó la necesidad de conservar el centro Histórico del departamento Jáchal, del norte de la Provincia de San Juan, ya que éste, por la historia provincial de condicionantes sísmicos y climáticos, se trata de uno de los sitios de la provincia que posee Patrimonio Histórico más antiguo y valioso. Reflejado en sus edificaciones coloniales que son un ejemplo singular de esta arquitectura en la provincia. Este proceso de inclusión del norte sanjuanino es una oportunidad invaluable para la
Palabras clave: centro histórico; patrimonio cultural; relevamiento; diagnóstico; planificación

\begin{abstract}
The present work belongs to the "Management Plan of the Historical Center of the city of Jáchal", San Juan, Argentina, for its conservation, revitalization, recovery and valorization. It was based on the survey of each plot of the historic center with pre-established forms, which took into account the most outstanding variables such as the type of construction, style, state, heritage value and land use. The stages were carried out in phases: in situ survey, data digitization and data interpretation grid.

As a final result, an analysis tool was obtained combining photographic urban profiles and the data of the historic center that enabled the corresponding diagnoses of the current situation of the analyzed area and to be able to make the normative guidelines for the conservation and valorization of the Center Historical of Jáchal.
\end{abstract}

Keywords: historical center; cultural heritage; survey; diagnosis; planning

\section{Introducción}

Salvador García Espinosa plantea como punto de partida la siguiente pregunta: Centros históricos ¿herencia del pasado o construcción del presente? $Y$ es a partir de ella que se plantea que no es posible estancar un centro en el tiempo, ya que el dinamismo de los procesos que influyen en un sitio son igual de valiosos que el sitio mismo. Por eso es necesario partir de que el concepto de Patrimonio se actualiza constantemente dando como resultado la identidad de una comunidad. Bajo esta premisa en un trabajo 
reactivación del área, abriéndola al exterior. Claro está que estos sitios con su carga cultural corren un verdadero peligro en contacto con el mundo exterior y el turismo. Sino se realizan las acciones necesarias para conservarlo, valorarlo y usarlo como recurso turístico sustentable en beneficio de la comunidad.

El Plan de manejo propuesto integra las áreas arquitectura, urbanismo y turismo. $Y$ el primer paso constó de la detección del patrimonio arquitectónico, para preservar su identidad y realizar acciones concretas de restauración, consolidación y puesta en valor.

Esto aportará al arraigo de la comunidad a su tierra evitando el éxodo masivo de jóvenes que comenzaran a ver en su lugar oportunidad de crecer. Es cierto que la conservación, en la antigüedad era antónimo del desarrollo de un sitio. Se parte entonces de la afirmación de que conservar el patrimonio cultural no significa estancar un sitio en el tiempo, por el contrario desde la arquitectura y la apropiación del espacio es que puede leerse los rasgos principales de la cultura de un sitio, destacando que la cultura está en constante evolución.

\section{Marco referencial}

El Departamento Jáchal se encuentra ubicado al norte de la provincia de San Juan, Argentina. Limita con los departamentos Caucete, Angaco, Albardón, Ullúm, Iglesia, Valle Fértil y la provincia de La Rioja. La cabecera departamental es la villa San José de Jáchal, ubicada a 157 kilómetros de la Capital de San Juan, con la que se comunica a través de la ruta nacional $N^{\circ}$ 40. La población se concentra en el Valle de Jáchal, el segundo en importancia económica y poblacional de la provincia.

La geomorfología del valle de Jáchal desempeña un rol determinante para los condicionantes climáticos. Se caracteriza por poseer un clima templado y seco. El departamento posee una gran variedad de recursos naturales que favorecen la principal actividad es la ganadería, pero en especial, el agro y en particular el cultivo de la cebolla ya que los suelos formados por arenas finas, limos y arcillas favorecen esta actividad. La explotación de minerales y rocas industriales también es de gran importancia. 


\section{San José de Jáchal}

Es la villa cabecera del departamento, se concentra la mayor proporción de población departamental. Se localiza entre la intersección de la Ruta Nacional N40 y la Ruta Nacional No150. Posee la mayor complejidad y nivel en todas las variables de la infraestructura de la población a escala departamental. Presenta una estructura urbana consolidada en damero.

Su apogeo fue desde mediados del siglo XIX a hasta principios del siglo $X X$, ocasionado por el esplendor minero. Esto produjo la construcción de importantes casonas construidas en adobe con estilo ecléctico italiano los cuales son considerados como palacios otorgándole una imagen particular y de valiosa identidad.

La traza fundacional de San José de Jáchal, que es de 1751, sigue intacta lo cual la hace muy valiosa mostrando un paisaje cultural que identifica a sus pobladores, posee un antiguo templo que es Monumento Nacional frente a su plaza principal, es una arquitectura modesta y majestuosa de adobe. El trazado de la localidad se caracteriza por tener calles anchas de la época anterior, enmarcadas con casonas características realizadas en tierra. Jáchal se considera como uno de los departamentos de la provincia donde aún se sigue manteniendo su patrimonio intangible como son sus costumbres, creencias y tradiciones, y en la conservación de muchas de las casonas e edificios industriales que poseen.

\section{Marco conceptual}

Resulta conveniente comenzar con la definición de dos conceptos básicos. Plan de manejo, Centros Históricos y Relevamiento. Un centro histórico no es otra cosa que el núcleo original de un área urbana que se caracteriza por contener en él, o haber contenido actividades de desarrollo de un área mayor además está caracterizado por la existencia en el de bienes urbanos, arquitectónicos y testigos intangibles vinculados al modo de vivir de esa comunidad y de su historia. En particular el centro histórico de Jáchal posee estas características. Por lo que se creyó de gran importancia la realización de un "Plan de manejo" que no es otra cosa que la planificación y regulación tendiente no solo a detener el accionar inescrupuloso de la actividad económica y sino también a valorar (como fin último) el sitio, pues reconocida en sí misma es que una comunidad puede relacionarse. Asegurando la Conservación, Valoración y Protección, de los sitios patrimoniales. 
Por consiguiente el primer paso obligado de este emprendimiento es conocer a través de un relevamiento exhaustivo cada uno de los rincones del centro histórico a conservar, por tal motivo el presente se evocará a esa primera y más importante etapa para luego determinar los lineamientos a seguir determinados por el diagnóstico.

\section{Método}

\section{RELEVAMIENTO}

Para el relevamiento se tomó como centro la plaza departamental y las 49 manzanas alrededor de la misma fijando como límites las calles Agustín Gómez al norte, General Acha al este, Aberastain al oeste y Laprida al sur. Las manzanas fueron denominadas con una letra mayúscula imprenta.

Procesamiento de datos: El proceso de relevamiento consto de diferentes fases:

\section{Relevamiento in situ}

El relevamiento propiamente dicho ejecutado mediante la realización de viajes al sitio con equipo de trabajo integrado por profesionales y estudiantes que realizaron exhaustivamente el levantamiento de datos en planillas confeccionadas para tal fin con pautas preestablecidas de llenado de las mismas, paralelamente se realizó el relevamiento fotográficotécnico mediante el cual se armaron perfiles por cuadra, los que permiten obtener la mayor exactitud posible del estado actual del casco histórico de Jáchal.

Este trabajo se realizó en base a planillas elaboradas por el equipo de trabajo (figura 1), y se le dio a los encuestadores una clase acerca del instructivo y criterio de llenado de las mismas. Las planillas fueron realizadas para registrar la mayor cantidad de datos relevantes posibles para luego poder abordar las estrategias convenientes en el plan de manejo. Cada una de las planillas posee la manzana correspondiente a relevar con su sistema parcelario e edilicio. Las mismas están divididas en distintos cuadrantes donde se colocaron los datos relevantes a tener en cuenta los cuales son Arquitectura que contiene el estilo de la construcción y el estado. El Valor particular donde se tienen en cuenta el tipo de construcción, el entorno morfológico, los aspectos característicos formales, el grado de adaptabilidad, el estado de conservación, la autenticidad y el grado de preservación. El cuadrante de Relevamiento urbano 
comprendido por el entorno circundante. El Valor patrimonial donde se tienen en cuenta los valores estéticos, históricos, social y ambiental. Y el Turismo donde se registra si el bien es un recurso turístico.

Digitalización de datos obtenidos por parte de cada relevador Para evitar inconvenientes el volcado de los datos a formato digital ser realizó por cada relevador, a fin de mantener el carácter primario de la información obtenida.

Grilla síntesis de análisis e interpretación de datos

En esta grilla se registró la síntesis del relevamiento de las planillas. Dividiendo al casco histórico en tres radios según su ubicación respecto a la plaza principal (figura 2). El primer radio se tomó por las cuadras que están alrededor de la plaza. El segundo radio son las primeras manzanas subsiguientes y el tercer radio lo comprenden las manzanas que encierran el casco.

Se utilizaron las variables más destacadas del relevamiento realizado las cuales fueron: Tipo de construcción, Estilo, Estado de la construcción, Valor patrimonial y Uso del suelo. Para luego poder volcar los datos en el plano del casco histórico y hacer los diagnósticos correspondientes de la situación actual de la zona analizada.

Esta grilla, permite visualizar el entrecruzamiento de variables y una vez finalizado el relevamiento permitirá seguir trabajando con los datos obtenidos, llegando a porcentajes generales y parciales de cada variable que se detallará más adelante, pudiendo traducir los datos en gráficos interpretativos (tipo torta o barra) además de facilitar la visualización de los datos.

Una vez obtenidos los datos se unificaron los datos obtenidos en el campo a través de la elaboración de una ficha síntesis comparativa que articula las variables más relevantes respecto a los objetivos de la primera etapa.

\section{Resultados}

\section{DIAGNÓSTICO}

Del análisis del relevamiento de la situación actual del casco histórico se vislumbraron las fortalezas y debilidades, oportunidades y amenazas. Mostrando y valorando el patrimonio cultural con que se cuenta, en qué condiciones se encuentra, los servicios e instalaciones turísticas básicas, los 
atractivos, en general se enumera a modo de ejemplo algunas de las situaciones que deberían existir para realizar la propuesta.

Variable: Tipo de construcción

En su mayoría las construcciones están realizadas en adobe, concentrándose en su en las zonas norte y oeste del casco. La zona sur se destaca por tener más construcciones tradicionales en el primer radio y manteniéndose en el segundo radio las construcciones de tierra. Alrededor de la plaza se aprecia una transformación de la construcción de adobe a la tradicional. La calle Juan de Echegaray se conforma como un recorrido donde se pueden apreciar las construcciones de tierra casi en su totalidad al igual que las calles Florida y Laprida.

Variable Estado de la construcción

Esta variable, muestra un estado constructivo bueno en general, aquí además de poder con gran facilidad sacar conclusiones del estado general del casco histórico podemos centrarnos en casos particulares, premisa tenida durante todo el procesamiento a fin de dejar sentada la posibilidad de un análisis pormenorizado.

Variable: Valor patrimonial

La valoración se realizó a partir de datos numéricos de calificación colocada por cada relevador en el sitio. Así se toman cuatro variables de valoración cada una con un rango de 0 a 4, se toma el valor histórico, social, estético y ambiental, a efectos de la síntesis para su interpretación y grafica se realiza la sumatoria de todas las variables estableciendo los siguientes rangos.

El centro histórico de Jáchal presenta un mayor número de construcciones con alto valor patrimonial en el primer radio frente a la plaza departamental. El valor va disminuyendo a medida que nos alejamos del centro histórico. Aunque existen manzanas dentro del tercer radio con alto valor patrimonial en su conjunto. En el cuadrante oeste (figuras 7 y 8). El centro histórico del departamento, único en la provincia en cuanto a su antigüedad y grado de originalidad debe ser protegido. Aquí se interpreta un fenómeno similar al ocurrido en la ciudad de San Juan que tiene que ver con una ciudad Intangible de un valor extraordinario, que subyace en lo que encontramos en la actualidad. Si se realzara un estudio evolutivo del centro histórico podríamos determinar como la puja inmobiliaria y el descreimiento del valor patrimonial van acabando con los testigos tangibles de la historia en de la provincia. 
Variable: Uso del suelo

En cuanto al uso del suelo los comercios se encuentran ubicados y concentrados en su mayoría alrededor de la plaza (primer radio) y en las manzanas circundantes (radio dos). Se destacan las calles San Juan y Juan de Echegaray como zonas comerciales ya que se extienden comercios casi en la totalidad de sus recorridos. A su vez en la zona norte del casco histórico prevalecen más comercios sucediendo lo contrario en la zona sur donde hay mayor concentración de uso residencial. El mismo se encuentra en el tercer radio. Existen varias zonas vacantes sobre todo al oeste de la plaza siendo de gran utilidad para futuros proyectos.

\section{Reglamento de preservación del pueblo histórico}

\section{GESTIÓN}

Urge proteger los bienes patrimoniales con normativas particulares para cada caso ya que las zonas rurales carecen de legislaciones particulares, y no siempre están contemplados en las leyes provinciales, por ejemplo en el caso de San Juan, los sistemas constructivos y materiales que predominan en las zonas rurales, no son aceptados por nuestras rigurosas leyes antisísmicas, lo cual genera un verdadero problema a tener en cuenta en las propuestas específicas en el patrimonio edificado.

Se requiere realizar gestiones ante los organismos responsables para elaborar reglamentaciones y leyes que protejan el patrimonio tangible e intangible.

El municipio de la Ciudad de Jáchal deberá ordenar su territorio en áreas rurales y área urbana, Definir un plan de mejoramiento de infraestructuras del centro de la ciudad. Para el área urbana deberá redactar un Plan Director que asegure:

-Un buen funcionamiento de los proyectos del Plan.

-La preservación de la trama de las 49 manzanas fundacionales, no permitiendo rupturas y/o despegues en las manzanas y el respeto por ambas líneas municipales.

-El respeto por las zonas de protección del patrimonio arquitectónico.

\section{ZONIFICACIÓN}

Se definió una zonificación compatible con la de conservación de los bienes patrimoniales. Se establecieron tres áreas de protección, con las 
cuales perfectamente se maneja el casco histórico con objetivos de conservación, preservación.

El Plan Director a nivel urbano se podría dividir según usos, población etc. en este caso para el plan de manejo se ha referido a los bienes patrimoniales de mayor valor, más vulnerables (construidas en adobe en su mayoría) y el estado de la construcción recordando que es este centro histórico el único de la provincia donde se conserva la imagen de distintas tipologías de viviendas con materiales locales.

\section{Área de protección 1}

Esta área se encuentra en el centro histórico, alrededor de la plaza principal, aquí encontramos construcciones de alto valor patrimonial construidas de adoben su mayoría. Se trata de casonas de fines del siglo XIX y principios del XX, que en la actualidad presentan uso mixto, es decir residencial y comercial. El estado de conservación de estas construcciones, en general es regular a deteriorado. En la actualidad se observa una tendencia importante al deterioro y reemplazo de casonas por construcciones nuevas de bajo valor. Frente a este fenómeno se considera que tiene un alto grado de protección por su valor histórico- ambiental de conjunto, además de encontrar dentro de este área construcciones de valor estético importante.

Sector alrededor de la plaza. La mayor concentración de edificaciones con valor histórico- social, y de mejor estado de conservación se encuentran frente a la plaza, por lo cual se establece un máximo grado de protección de estas edificaciones, con el objetivo de conservar la imagen urbana actual y revertir el proceso de cambio y desvalorización de las construcciones de adobe.

Así mismo se plantea la necesidad de tener en cuenta el tipo de uso y recurrencia de personas en construcciones de adobe, estableciendo reglamentación singular a cada caso respecto a la estabilidad.

Sector periférico. El sector periférico presenta un menor valor patrimonial, recomendando un grado de protección medio que permita un grado de adaptabilidad mayor y recomendando su protección para la conservar el entorno morfológico- ambiental. 


\section{Área de protección 2}

Se trata del noroeste del centro histórico de la ciudad de Jáchal, aquí se observan la mayor concentración de construcciones de adobe, de buen estado de conservación predominando el uso residencial en casonas históricas, imperando el valor patrimonial medio a bajo. Se propone un grado de protección medio que permita adaptaciones en construcciones de bajo valor, conservando el entorno morfológico respecto a las alturas de las construcciones, retiros y características urbanas residenciales del siglo XIX.

Se encuentran dentro de esta área también casos particulares de alto valor e importancia, para los cuales se proponen que integren al área de protección 1.

\section{Área de protección 3}

Se trata del sureste del centro histórico de uso netamente residencial con la mayoría de las construcciones de adobe con bajo valor patrimonial, es un área que se caracteriza por un alto grado de transformación de las construcciones sobre todo en el área cercana a la plaza principal. Se propone:

- Un grado de adaptabilidad alto, se requiere la conservación del entorno morfológico respecto a las alturas de las construcciones, retiros y características urbanas residenciales del siglo XIX y XX. Estas sub áreas tendrán siempre una fluida vinculación e integración procurando un desplazamiento seguro de vehículos y peatones diferenciándose entre ellos.

- Definir un Modelo de Capacidad de Carga: (establecer cuál es el número de visitantes que puede soportar, simultáneamente, el área central del casco histórico), la aplicación de un modelo de capacidad de carga es fundamental para evitar los impactos generados por la visitación de los turistas.

- Se deberá realizar la elaboración y aplicación de un modelo que mida los efectos ambientales Estudio de Impacto Ambiental (Monitoreo), ya que es fundamental para un plan de manejo. Generados por los visitantes y los impactos que produce en las áreas de conservación, se circunscriben a las zonas de uso público.

- Para un adecuado manejo turístico patrimonial sostenible hay que integrar a este las comunidades periféricas al área de conservación, especialmente las que se encuentran en la zona central. El proceso 
de integración tiene por objetivo la valoración del área por la comunidad, su participación en el turismo, en la conservación, en la mejoría de su calidad de vida, entre otros factores para tender al desarrollo sostenible, y la ejecución de los proyectos de capacitación y concienciación, entre otros.

- Regulaciones y normas que tengan como finalidad establecer controles a todas las actividades que se realicen en los bienes patrimoniales. Monitoreo de las obras a cargo de especialistas para asegurar los principios que la conservación patrimonial impone. Se deberá realizar un Análisis Económico tomando en consideración los costos y beneficios.

- Trabajar con la gestión mixta: lo público y lo privado, en forma transversal con otras áreas de gobierno. Proceso continuo de participación e interconsulta permanente entre municipio y los referentes de la comunidad del proyecto a la obra. La participación civil.

- Gestionar las fuentes de financiamiento y esponsoreo de empresas privadas, para las inversiones requeridas en todo plan de manejo.

\section{Lineamientos normativos: urbanismo y arquitectura}

Lineamientos tecnológicos

Proyectar y construir pensando en el uso de materiales naturales propios de la zona y con tecnologías apropiadas.

Intervenir en las construcciones contemporáneas, con técnicas y materiales que mantengan un equilibrio de expresión, apariencia, textura y forma con la estructura original, reforzando las estructuras con tecnologías modernas.

En el caso de cambiar y/o adaptar los usos, como transformar en comercios o confiterías o bares las casonas se deberán estudiar su Estabilidad: Uso adecuado de contrafuertes para mejorar la estabilidad de los muros en los encuentros exteriores de los muros la longitud mínima debe ser igual al espesor de muro. Limites de altura y longitud de los muros: la altura de los muros debe ser ocho espesores del muro y la longitud máxima de los muros diez espesores de muro.

Lineamientos morfológicos

Adecuar las nuevas edificaciones o las remodelaciones de las no tradicionales al carácter estético de la tipología y acabados tradicionales. 
Mantener la alineación y tamaño de aberturas, alturas y proporciones.

Lineamientos funcionales

Compatibilizar los nuevos usos (almacenes, hostales, comercios, etc.) con la arquitectura tradicional de la zona.

\section{Lineamientos ambientales}

Elaboración de recomendaciones en la construcción de acuerdo al entorno.

Respetar, apreciar y tener en cuenta la correcta percepción y dignidad de la escena o paisaje en las actuaciones nuevas.

Mantener la parcelación y mantener las alineaciones históricas de la edificación.

Conseguir buena relación con el entorno y el paisaje en el diseño arquitectónico.

Respetar el modo de ocupación del sitio, la relación del edificio con la calle y la conformación del tejido urbano.

Patrimonio intangible

Rescatar las técnicas de construcción con tierra, de los pobladores locales e intercambiar con nuevas experiencias tecnológicas.

Promover técnicas de tejidos a telar, y artesanías varias.

Difundir en almanaque turístico fiestas populares y religiosas.

Dictar talleres de danzas, artesanías y gastronomía.

\section{Publicidad}

Redactar un código de publicidad urbano (ordenanza municipal) que regule La actividad publicitaria (toda acción tendiente a dar a conocer una labor o un producto comercial industrial, o de servicio público o privado, realizado en La vía pública y / o sitios de accesos o vista al público a través de medios empleados para el cumplimiento de sus fines).

Los elementos publicitarios (avisos, letreros, volantes luminosos electrónicos banners, gazebos vidrieras etc.) deberán estar regulados en tamaño tipografías etc. 
No podrán ser frontales sobre superficies de adobe y tampoco sobresalir de las medidas establecidas. Si Forman parte de La fachada deberán armonizar con su arquitectura respeta

\section{Conclusión}

Este relevamiento resultó ser una herramienta de mucha utilidad y necesaria ya que fue la columna vertebral para poder saber en qué estado se encuentra la situación actual del casco histórico. Así se pudieron en base a éste, tomar decisiones coherentes para realizar los lineamientos estratégicos en cuanto a la conservación, revitalización, recuperación y puesta en valor. Ya que dentro de estas edificaciones se contienen mensajes inmateriales, que forman su espíritu y su identidad, que se contiene en el contexto cultural donde están insertos, trascendiendo su historia y otorgándole un alto grado de pertenencia.

El Plan de puesta en valor del antiguo pueblo de Jáchal, especifica la necesidad de dar un impulso al desarrollo de la Ciudad de como cabecera del oasis y replicar las acciones en el resto del departamento. Como también trasladar la experiencia a los departamentos de Iglesia y Valle Fértil unidos por el tajo territorial bituminoso de la ruta nacional 150, futuro Corredor Andino.

El Programa de renovación urbana de la ciudad, centrado en la cultura, constituye el pilar del desarrollo económico, social y cultural de la ciudad de Jáchal, y viene desarrollándose gestiones y acciones desde el diseño y la cultura poética Jachallera.

El objetivo es dar una mirada desde afuera para ayudar a los gestores a reconocer cuales serían las metas a perseguir y pensar cómo debería ser un Jáchal moderno desde la cultura y tradición. 


\section{BIBLIOGRAFÍA}

Bayardo, Rubens, Lacarrie, Mónica. (2003). Globalización e identidad cultural. Buenos Aires. Editorial: CICCUS.

De Paula, Alberto. (2006). La esencia social de la cultura y el patrimonio. Autenticidad = Identidad. Actas del VIII Congreso de Rehabilitación del Patrimonio Arquitectónico y Edificación. Dimensión Social del Patrimonio. Salta. Argentina.

Fernández Boan, Alicia, y Alfaro, Alberto. (2008). Principios y técnicas de Conservación. Patrimonio Arquitectónico Argentino 1850-1950. Buenos Aires. Editorial: Hábitat.

Foglia, María Elena. (1992). Rehabilitación de los pequeños poblados históricos. Un enfoque regional para la rehabilitación y desarrollo de los poblados históricos. 1er. Curso Internacional de Especialización. Facultad de Arquitectura. Universidad Nacional de Asunción. Paraguay.

Gordillo, Sandra et al. (2003). Fragmentos para una identidad. Actitudes para afrontar la crisis ambiental y valorar el patrimonio. Universidad Nacional de Córdoba. Argentina.

ICOMOS. (1999). Carta del Patrimonio Vernáculo construido. México.

Moreno, Carlos et al. (1995). De las viejas tapias y ladrillos. Españoles y criollos, largas historias de amores y desamores. Buenos Aires. Editorial: Centro para la Conservación del Patrimonio Urbano y Rural.

Peñaloza De Varese, Carmen y Arias, Héctor D. (1964). Historia de San Juan. San Juan. Editorial Spadoni.

Plana, María Rosa, Orellano, Luis. (2006). Rescate de tipologías como aporte al diseño. Patrimonio Vernáculo Iglesiano. SIACOT. Mendoza. Argentina.

Plana, María Rosa, Fábrega, Mabel et al. (2007). El patrimonio cultural y natural del departamento Iglesia como elemento articulador para el desarrollo local. Proyecto de Investigación. CICITCA- UNSJ, San Juan. 
Suárez Inclan, María Rosa. (2002). Los Itinerarios Culturales. The CllC Scientific Magazzine.

UNESCO. (1994). Documento de Nara sobre Autenticidad. Tailandia.

Viñuales, Graciela María. (1981). Restauración de Arquitectura de Tierra. Tucumán. Editorial: Instituto Argentino de Investigaciones de Historia de La Arquitectura del Urbanismo. 
